SESSION 3: Spectral energy distribution models 


\title{
A review of spectral energy distribution modeling at high-redshift
}

\section{Stéphane Charlot}

Institut d'Astrophysique de Paris, France

\begin{abstract}
I will review recent developments in the modeling of high-redshift galaxy spectra, focusing in particular on the rest-frame ultraviolet and optical emission from young stellar populations and the interstellar medium.
\end{abstract}

